\title{
Gaps in life expectancy for people with type 1 diabetes
}

\author{
Lars C. Stene ${ }^{1,2}$
}

Received: 4 March 2016 / Accepted: 7 March 2016/Published online: 5 April 2016

(C) Springer-Verlag Berlin Heidelberg 2016

\begin{abstract}
Two papers in this issue of Diabetologia present recent trends in life expectancy for people with type 1 diabetes, one using data from an Australian registry (Huo et al, DOI: $10.1007 / \mathrm{s} 00125-015-3857-4)$, the other, a Swedish registry (Petrie et al, DOI: 10.1007/s00125-016-3914-7). This commentary provides a brief review of the concept of the period life expectancy and complexities regarding applicability to patients, before summarising and discussing the main results of the two papers. In addition, some remaining relevant knowledge gaps are discussed.
\end{abstract}

Keywords Clinical · Clinical epidemiology $\cdot$ Epidemiology Human $\cdot$ Life expectancy $\cdot$ Mortality $\cdot$ Registries $\cdot$ Type 1 diabetes

Despite tremendous improvements in the prognosis for patients with type 1 diabetes since the 1920s, significant gaps in mortality compared with the general population remain [1-4]. In addition to monitoring other quality measures of the care and wellbeing of patients [5], it is of interest to document in population-based samples whether the mortality gap has diminished in recent years. In this issue of Diabetologia, Petrie et al [6], and Huo et al [7], present recent trends in life expectancy for people with type 1 diabetes.

Contrary to the life expectancy estimated in cohorts observed for the entire lifespan, which have mostly historical

Lars C. Stene

Lars.Christian.Stene@fhi.no

1 Norwegian Institute of Public Health, P.O. Box 4404 Nydalen, NO-0403 Oslo, Norway

2 Oslo Diabetes Research Centre, Oslo University Hospital, Oslo, Norway interest, the period life expectancy is a useful and summary measure of 'current' population health [8]. It is estimated from the mortality experience across all ages during a short period such as a single calendar year, and therefore pertains to hypothetical individuals who experience each of the age-specific mortality rates observed in the current period. The old saying that it is difficult to make predictions, especially about the future, is also valid for the period life expectancy. If not a naive one, it is not really a prediction. To estimate life expectancy, we need mortality rates across all age groups. The estimated life expectancy does not contain more information than the mortality rates on which it is based, but it provides a different perspective that may be useful.

Despite the immediate appeal to patients, clinicians and policy makers, interpretation and application of life expectancy for patients with type 1 diabetes is not straightforward. Life expectancy at birth for people with type 1 diabetes is difficult to grasp, because patients are diagnosed at various ages, years or decades after birth. As a population summary metric, it provides integrated information about the age-specific mortalities, but it does not pertain to a single patient. One should always be careful when applying study results to individual patients, but what matters for clinicians and patients is the estimates of remaining life expectancy from now. This requires estimates of remaining life expectancy, conditional on survival up to this point. If a patient at a given time (now) has long-standing disease, the relevant life expectancy should ideally be based on data from other patients with similar disease durations.

With these complexities in mind, what do these new studies tell us? Both studies used nationwide registers that had been collecting data since 1987. The Swedish study [6] estimated remaining life expectancy at age 20 years (for the periods 2002-2006 and 2007-2011), and the Australian study [7] estimated remaining life expectancy for different age groups 
(for the periods 1997-2003 and 2004-2010). For comparison between the studies, life expectancy at age 20 years in the Australian study can be approximated by the mean of results for the groups 15-19 and 20-24 years (presented in ESM Table 2 of the paper [7]). For the most recent periods among men the remaining life expectancy at age 20 was 49.7 in Sweden and 50.8 years in Australia, and for women the estimate was 51.9 years in Sweden and 54.3 in Australia. Compared with a previous study from Scotland covering the period 2008-2010, the estimates from Sweden and Australia were all higher by approximately 3.5-6 years [9].

The difference in life expectancy at age 20 among patients with type 1 diabetes and the respective background populations ('loss of life expectancy', 'years of life lost' or 'gap in life expectancy') were approximately 10-13 years both in Sweden, Australia (and Scotland [9]), with slight differences between studies. The increase in remaining life expectancy over the 5-7 year periods, approximately 2 years for men, 1.3 years for Australian women, and essentially zero for Swedish women, were very similar to the increase in the corresponding general populations during the same periods. It therefore seems that the gap in life expectancy has remained largely unchanged since the turn of the millennium.

On the one hand, the studies by Petrie et al and Huo et al were based on approximately 30,000 and 85,000 patients, respectively, and should be powered to detect small increases in life expectancy in recent years. On the other hand, it is perhaps not surprising that the gap in life expectancy has remained largely unchanged in recent years, because the differences in lifetime exposure to hyperglycaemia and other determinants of survival in the two overlapping groups of people with type 1 diabetes examined for mortality during these recent years may not be very different. We know that glycaemic control has long lasting effects [10,11]. Over the past decades, there have been increases in life expectancy in the general population of Sweden, Australia and most other countries of the world [6-8], in part because of a remarkable reduction in cardiovascular mortality [12]. Cardiovascular risk management is an integral part of diabetes care, and it is likely that patients with type 1 diabetes have enjoyed some of the beneficial developments that do not involve glycaemic control alone.

There is still a knowledge gap regarding life expectancy for patients with type 1 diabetes. Duration of disease is often predictive of survival, but is usually ignored in studies of life expectancy because of lack of data. What matters for a newly diagnosed patient is the relevant estimates of mortality and relative mortality based on newly diagnosed patients, ideally diagnosed at a similar age. On the other hand, life expectancy estimated from mortality in newly diagnosed patients will necessarily have to be based on patients diagnosed at various ages, and thus cannot apply to any individual patient (since all individual patients are diagnosed at a single age). We still lack good data on life expectancy for patients diagnosed with type
1 diabetes at 50 years of age or older. The obvious reason for this lack of knowledge is the difficulty of classification of diabetes as type 1 vs type 2 in the higher age groups, and lack of detailed clinical information in most population-based registers of sizes that allow accurate estimation of life expectancy. In the studies presented in the this issue, patients had to be $<30$ years [6] or $<45$ years [7] at diagnosis. This means that all observed mortality in the oldest age groups were based on patients who had to survive for decades after diagnosis to contribute to the studies. For example, an 80 -year-old Swedish patient who died in 2010 had been diagnosed before 1960 and would have survived for 50 years thereafter. While the statistical analysis of such so-called left-truncated data takes this into account by following patients from study entry rather than from diagnosis, we still do not know anything about the mortality or survival in the first years after diagnosis of type 1 diabetes in those diagnosed in the highest age groups.

In summary, Petrie et al and Huo et al used impressively large population-based registries to estimate life expectancy for patients with type 1 diabetes, and documented a gap compared with the general population that has not diminished materially over recent years. Some knowledge gaps have been filled, but others remain.

Acknowledgements The author would like to thank colleagues K. Bævre at the Norwegian Institute of Public Health, and G. Joner at Oslo University Hospital, for comments on earlier versions of the manuscript.

Funding The author is supported by the Research Council of Norway.

Duality of interest The author declares that there is no duality of interest associated with this manuscript.

Contribution statement The author was the sole contributor to this paper.

\section{References}

1. Morgan E, Cardwell CR, Black CJ, McCance DR, Patterson CC (2015) Excess mortality in type 1 diabetes diagnosed in childhood and adolescence: a systematic review of population-based cohorts. Acta Diabetol 52:801-807

2. Lind M, Svensson AM, Kosiborod M et al (2014) Glycemic control and excess mortality in type 1 diabetes. N Engl J Med 371:19721982

3. Harding JL, Shaw JE, Peeters A, Guiver T, Davidson S, Magliano DJ (2014) Mortality trends among people with type 1 and type 2 diabetes in Australia: 1997-2010. Diabetes Care 37:2579-2586

4. Gagnum V, Stene LC, Sandvik L et al (2015) All-cause mortality in a nationwide cohort of childhood-onset diabetes in Norway 19732013. Diabetologia 58:1779-1786

5. American Diabetes Association (2016) Standards of medical care in diabetes-2016. Diabetes Care 39(Suppl 1):S1-S109 
6. Petrie D, Lung T, Rawshani A et al (2016) Recent trends in life expectancy for people with type 1 diabetes in Sweden. Diabetologia. doi:10.1007/s00125-016-3914-7

7. Huo L, Harding JL, Peeters A, Shaw JE, Magliano DJ (2016) Life expectancy of people with type 1 diabetes in 1997-2010: a national Autralian registry-based cohort study. Diabetologia. doi:10.1007/ s00125-015-3857-4

8. Global Burden of Disease 2013 Study Mortality and Causes of Death Collaborators (2013) Global, regional, and national age-sex specific all-cause and cause-specific mortality for 240 causes of death, 1990-2013: a systematic analysis for the Global Burden of Disease Study 2013. Lancet 2015;385:117-171
9. Livingstone SJ, Levin D, Looker HC et al (2015) Estimated life expectancy in a Scottish cohort with type 1 diabetes, 2008-2010. JAMA 313:37-44

10. Orchard TJ, Nathan DM, Zinman B et al (2015) Association between 7 years of intensive treatment of type 1 diabetes and longterm mortality. JAMA 313:45-53

11. Chiang JL, Kirkman MS, Laffel LM, Peters AL (2014) Type 1 diabetes through the life span: a position statement of the American Diabetes Association. Diabetes Care 37:2034-2054

12. Ezzati M, Obermeyer Z, Tzoulaki I, Mayosi BM, Elliott P, Leon DA (2015) Contributions of risk factors and medical care to cardiovascular mortality trends. Nat Rev Cardiol 12:508-530 